\title{
Ce valence in intermetallic compounds by means of XANES spectroscopy
}

\author{
Alexander Yaroslavtsev*,I, Alexey Menushenkovi, Roman Chernikov ${ }^{\text {I }}$, Evgeny Clementyev ${ }^{\text {II }}$, Vladimir Lazukov ${ }^{\text {III }}$, \\ Yan Zubavichus ${ }^{\mathrm{III}}$, Alexey Veligzhanin ${ }^{\mathrm{III}}$, Nadejda Efremova ${ }^{\mathrm{III}}$, Alexander Gribanov ${ }^{\mathrm{IV}}$ and Anatoly Kuchin ${ }^{\mathrm{V}}$ \\ I National Research Nuclear University "MEPhI", Kashirskoe sh., 31, 115409 Moscow, Russia \\ II Institute for Nuclear Research RAS, 60-letiya Oktyabrya prospekt, 7a, 117312 Moscow, Russia \\ III Russian Research Centre "Kurchatov Institute", Academic Kurchatov sq., 1, 123182 Moscow, Russia \\ IV Chemistry Department, Lomonosov Moscow State University, Leninskie Gory, GSP-1, 119991 Moscow, Russia \\ v Institute of Metal Physics UB RAS, S. Kovalevskoy, 18, 620990 Ekaterinburg, Russia
}

Received July 19, 2010; accepted August 9, 2010

\section{Cerium / Valence / XANES / Rare-earth / Intermetallics}

\begin{abstract}
The cerium effective valence was investigated by means of $\mathrm{Ce} L_{3}$-XANES spectroscopy in some intermediate valence Ce-based systems with different magnetic ordering mechanisms: $\mathrm{CeNi}$ doped with $\mathrm{Nd}, \mathrm{Pr}$ and $\mathrm{Gd}$ in the temperature range 5-300 $\mathrm{K}$, and $\mathrm{Ce}_{2} \mathrm{Fe}_{17-x} \mathrm{Mn}_{x}$ intermetallics. The dependences obtained are considered within the frames of generally accepted mechanisms of rare-earth ions intermediate valence states. Possible correlations between the cerium effective valence and the magnetic properties of compounds are also noted.
\end{abstract}

\section{Introduction}

In rare-earth $(\mathrm{Ce}, \mathrm{Sm}, \mathrm{Eu}, \mathrm{Tm}, \mathrm{Yb})$ compounds the $f$-electrons are characterized by a small binding energy, which causes their transfer into the conduction band. But the strong on-site Coulomb interaction prevents this transfer. Counteraction of the opposite trends to localization and delocalization of $f$-electrons in rare-earth compounds leads to a high density of states near the Fermi level and to a great variety of physical effects. There are intermediate valence states (IV), the Kondo effect, the heavy fermion state and different kinds of electron instabilities among them. These properties are in strong dependence of the chemical environment, of external pressure and temperature.

In this work the cerium effective valence was investigated by means of $L_{3}$-XANES spectroscopy in some intermediate-valence Ce-based systems with different magnetic ordering mechanisms: $\mathrm{CeNi}$ doped with $\mathrm{Nd}, \mathrm{Pr}, \mathrm{Gd}$, and also $\mathrm{Ce}_{2} \mathrm{Fe}_{17-x} \mathrm{Mn}_{x}$ intermetallics. $L_{3}$-XANES spectroscopy is based on the electron excitation from the $2 p^{3 / 2}$ core level to the $5 d$ - valence orbital. The increase of the valence shifts the absorption edge position to higher energy. In the intermediate valence compounds the peak in

* Correspondence author (e-mail: yalex03@gmail.com) the absorption spectrum is split because of the two-way electron excitation, corresponding to the different valence states. The characteristic time of fluctuations between these two states is about $10^{-13}-10^{-14}$ sec. [1]

As it is assumed nowadays, the intermediate-valence state of $\mathrm{Ce}$ in intermetallic compounds with $d$-metals is not specified by a $4 f \rightarrow 5 d$ electron transfer like in other unstable valence lanthanides, but more likely by delocalization of the $4 f$ states itself due to $4 f$ wave functions overlap in the different sites [2]. Hybridization between the conduction band and the $4 f$-electrons and hence the rare-earth effective valence increase are usually caused by applying either external or "chemical" pressure to the intermediate valence compound. The latter so called crystalchemical compression is usually achieved by the substitution of the initial rare-earth with an ion of smaller radius. At the same time, the value of valence itself is defined by another mechanism. In compounds with $3 d$ metals the presence of an ionic component in the interatomic interaction might influence the rare-earth valence [3, 4]. It depends both on the difference between the rare-earth and the $3 d$ element donor and acceptor properties, such as electronegativity, and the compound's structural peculiarities, such as the number of $3 d$ atoms in the rare-earth environment and the interatomic distances. Besides, the CeNi-based compounds are subject to the hydrogenation which causes the formation of various hydrides $\left(\mathrm{CeNiH}_{x}, \mathrm{CeH}_{x}\right.$, etc. $)$ as well as other $\mathrm{CeNi}_{x}$ phases, and stabilizes the $4 f^{1}$ or trivalent Ce state [5].

The recent experiments on pseudo-binary non-magnetic CeNi-based compounds with Ce substitutions by Gd and Pr showed that the partial delocalization of the cerium $4 f$ electrons led to a significant increase of the Curie temperature for the $\operatorname{Pr}$ and $\mathrm{Gd}$ substructures [6,7]. It is known that Kondo interactions and indirect exchange interactions (RKKY) are general competitors in formation of magnetic or nonmagnetic basic states in the strongly correlated electronic systems. However, Kondo interactions can cause the magnetic ordering in compounds with coexisting subsystems of ions with a valence instability and rare- 
earth ions with well localized magnetic moments. Concerning another type of systems, in which a cooperation between the rare-earth ions with intermediate valence state and the $3 d$ elements takes place like in our $\mathrm{Ce}_{2} \mathrm{Fe}_{17}$ compound, it is assumed that the type of magnetic state and Curie temperature result from the competition of positive and negative exchange interactions between the $3 d$ metal atoms [8]. The phase diagram of $\mathrm{Ce}_{2} \mathrm{Fe}_{17-x} \mathrm{Mn}_{x}$ is rather complicated: the basic magnetic state is ferromagnetic, but in the $\mathrm{Mn}$ concentration range $x=0.5-1$ it is helical antiferromagnetic [9]. One reason for the non-monotonous dependence of the magnetic parameters can be an instability of the Ce atom valence state: the Ce $4 f$ electronic state throughout its hybridization with $3 d$-electrons of $\mathrm{Fe}$ and Mn can influence the magnetic state of the whole compound. This is one of the problems the present work is also intended to clarify.

\section{Experimental}

$\mathrm{Gd}_{x} \mathrm{Ce}_{1-x} \mathrm{Ni} \quad(x=0.05,0.15,0.50,0.85), \quad \operatorname{Pr}_{x} \mathrm{Ce}_{1-x} \mathrm{Ni}$ $(x=0.25,0.85)$ and $\mathrm{Nd}_{x} \mathrm{Ce}_{1-x} \mathrm{Ni}(x=0.25,0.50,0.80)$ samples were prepared by arc melting of high purity elements $(\sim 99.98)$ in an argon atmosphere. The obtained compositions were then recrystallized in vacuum and founded in cylindrical ingots of a diameter $\sim 10 \mathrm{~mm}$. The ingots were then annealed in dynamical vacuum at $600{ }^{\circ} \mathrm{C}$ for $150 \mathrm{~h}$. Finally, to prevent the hydrogenation and oxidation in the water vapor of the air, the samples were kept in the helium glovebox until the time of experiment. $\mathrm{Ce}_{2} \mathrm{Fe}_{17-x} \mathrm{Mn}_{x}$ samples $(x=0.0,0.5,1.0,1.3,2.0)$ were prepared by argon arc melting from the starting materials. The ingots were homogenized at $1450 \mathrm{~K}$ for $9 \mathrm{~h}$ and then quenched in water. To check the purity the X-ray diffraction experiments were carried out on the powder samples at room temperature on the diffractometers STADI MP and DRON UM1 with $\mathrm{Cu} K_{\alpha}$-radiation. No extraneous phases were detected in the CeNi-based samples. Whereas, all the $\mathrm{Ce}_{2} \mathrm{Fe}_{17}$-based samples have the 2:17 phase and a small amount of the undissolved $\alpha$-Fe less then $4 \%$.

$L_{3}$-absorption spectra $\left(L_{3}\right.$-XANES) of the Ce compounds $(\mathrm{Gd}, \mathrm{Pr}, \mathrm{Nd})_{x} \mathrm{Ce}_{1-x} \mathrm{Ni}$ were collected at beamlines $\mathrm{E} 4$ and $\mathrm{C}$ of the synchrotron center HASYLAB, DESY (Germany, Hamburg), in the total fluorescence yield mode at different temperatures in the range 5-300 K. Additional data were measured at the KMC-2 beamline of BESSY II (Germany, Berlin). Spectra of the $\mathrm{Ce}_{2} \mathrm{Fe}_{17-x} \mathrm{Mn}_{x}$ samples were collected at room temperature at the experimental station "Structural Materials Science" of KCSR and NT (Russia, Moscow) in transmission mode.

\section{Results and discussion}

The normalized $\mathrm{Ce}_{3}$ edge steps of the $\mathrm{Gd}_{x} \mathrm{Ce}_{1-x} \mathrm{Ni} \mathrm{X}$-ray absorption spectra of compounds with maximal $(x=0.05)$ and minimal $(x=0.85)$ Gd concentrations obtained at room temperature are presented in Fig. 1. All spectra obviously contain one strong peak $\mathrm{A}$ at an energy $\sim 5723.5 \mathrm{eV}$ that corresponds well to the $\mathrm{Ce}_{3}$ absorption edge for the

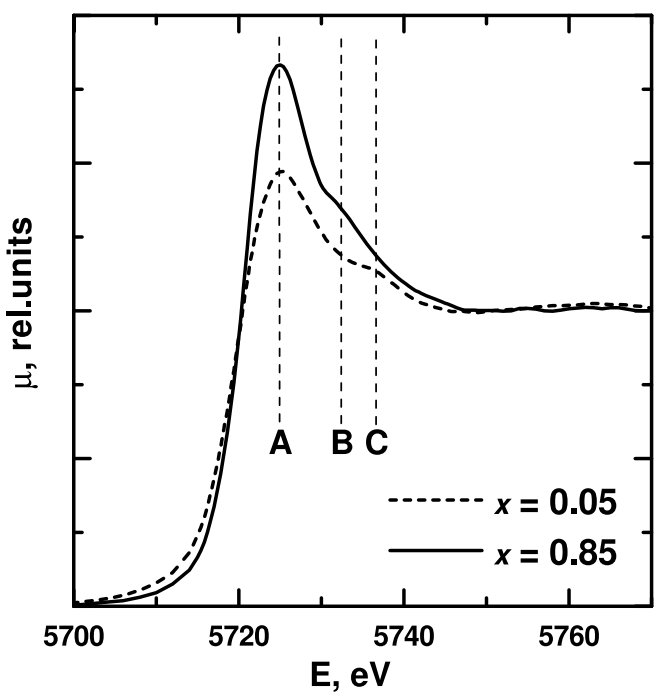

Fig. 1. $\mathrm{Ce} L_{3}$ absorption spectra of $\mathrm{Gd}_{0.05} \mathrm{Ce}_{0.95} \mathrm{Ni}$ and $\mathrm{Gd}_{0.85} \mathrm{Ce}_{0.15} \mathrm{Ni}$ at room temperature. Peak $\mathrm{A}-\mathrm{Ce}^{3+}$ contribution; peak $\mathrm{B}-\mathrm{Ce}^{4+}$ contribution; peak $\mathrm{C}$ probably is the result of photoelectron multiple scattering on the Ce local environment.

trivalent electronic configuration. The $\mathrm{Ce}^{3+}$ and $\mathrm{Ce}^{4+}$ contributions are almost unresolvable because the tetravalent component is very weak. The amplitude of the main peak increases with the Gd concentration and the partially resolvable component $\mathrm{B}$ appears at an energy of $\sim 5732 \mathrm{eV}$ corresponding to the $\mathrm{Ce}^{4+}$ contribution. The unidentified week peak $\mathrm{C}$ at $\sim 5735 \mathrm{eV}$ is the best resolved in the spectrum of $\mathrm{Gd}_{0.05} \mathrm{Ce}_{0.95} \mathrm{Ni}$ as in spectra of all CeNi-based compounds with a small dopant concentration. Since the $\mathrm{Ce}^{4+}$ contribution is very weak, it overlaps with peak $\mathrm{C}$, which makes precise spectra decomposition difficult. Besides, the amplitudes of all CeNi-based spectra are affected by the self-absorption to a variable degree. This is due to the samples specific character and fluorescent measurement mode but should not affect the ratio of different valence components. $\mathrm{Ce}_{3}$-XANES spectra of few $\mathrm{Ce}_{2} \mathrm{Fe}_{17-x} \mathrm{Mn}_{x}$ samples are shown in Fig. 2. Hear both the $\mathrm{Ce}^{3+}$ and $\mathrm{Ce}^{4+}$ components are clearly resolved, which apparently comes from the greater tetravalent contribution.

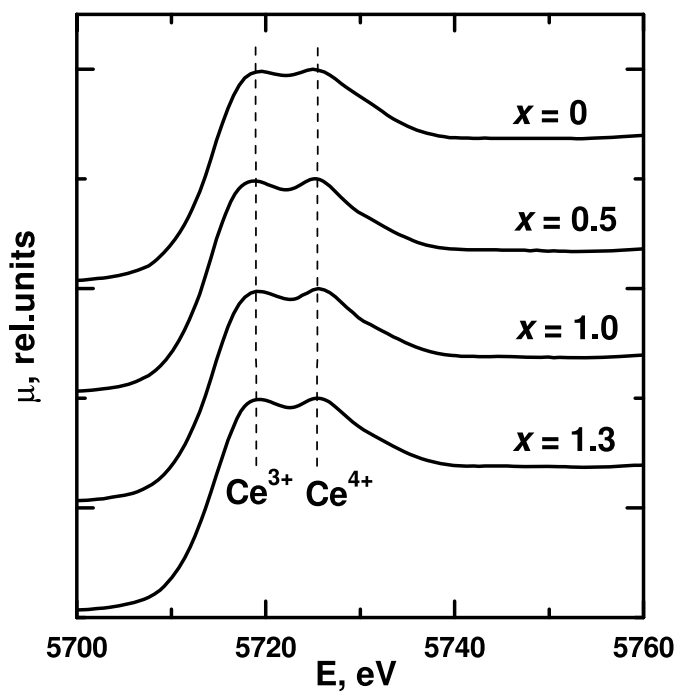

Fig. 2. $\mathrm{Ce} L_{3}$ absorption spectra of various $\mathrm{Ce}_{2} \mathrm{Fe}_{17-x} \mathrm{Mn}_{x}$ samples at room temperature. $\mathrm{Ce}^{3+}$ and $\mathrm{Ce}^{4+}$ components are clearly resolved. 
Unlike the CeNi-based spectra, $\mathrm{Ce}_{2} \mathrm{Fe}_{17-x} \mathrm{Mn}_{x}$ do not demonstrate the third contribution. Additional calculations of the absorption step with the FEFF8.20 code [10] have shown that this spectral feature is caused by the effects of photoelectron multiple scattering in crystalline environments of $\mathrm{Ce}$, which are obviously different in two compounds. Such effects are significant just in the near edge region of XAFS spectra.

In the present work the extraction of different cerium valence components and evaluation of their contribution into the $L_{3}$-XANES spectrum was performed using the conventional fitting of complicated "white lines" with combinations of Lorentzian and arctangent curves of constrained widths and energy positions [11] after the polynomial background removal from the experimental spectrum. The analytic functions obtained were convoluted with the Gaussian of width $1.60-2.50 \mathrm{eV}$ to take into account the additional experimental broadening. In this approach the amount of $\mathrm{Ce}$ ions of each valence state is assumed to be proportional to the area under the corresponding Lorentzian curve. The example of the absorption peak decomposition into the $\mathrm{Ce}^{3+}$ and $\mathrm{Ce}^{4+}$ components for $\mathrm{Gd}_{0.85} \mathrm{Ce}_{0.15} \mathrm{Ni}$ is shown in Fig. 3. The amplitude of the tetravalent component is a few times lower than that of the trivalent one. Also note, how broad both components are.

The total width of each valence component turned out to be $\sim 5.5 \mathrm{eV}$, where $\sim 3.5 \mathrm{eV}$ is the natural width of $L_{3}$ level [12] and $\sim 2 \mathrm{eV}$ is the experimental broadening. The energy gap between the two components is $\sim 9 \mathrm{eV}$. Therefore, peaks overlap strongly and unambiguous extraction of two spectral contributions is rather difficult. To solve this problem several decompositions of each spectrum were made with slight variations of the fitting parameters, such as Lorentzian or Gaussian widths, a gap between two components, etc. Thus, statistics were collected to refine the valence values. This technique was especially useful for resolving the CeNi-based spectra since the tetravalent component in these compounds is quite weak.

The concentration dependences of the cerium effective valence in the CeNi-based compounds (Fig. 4) are in

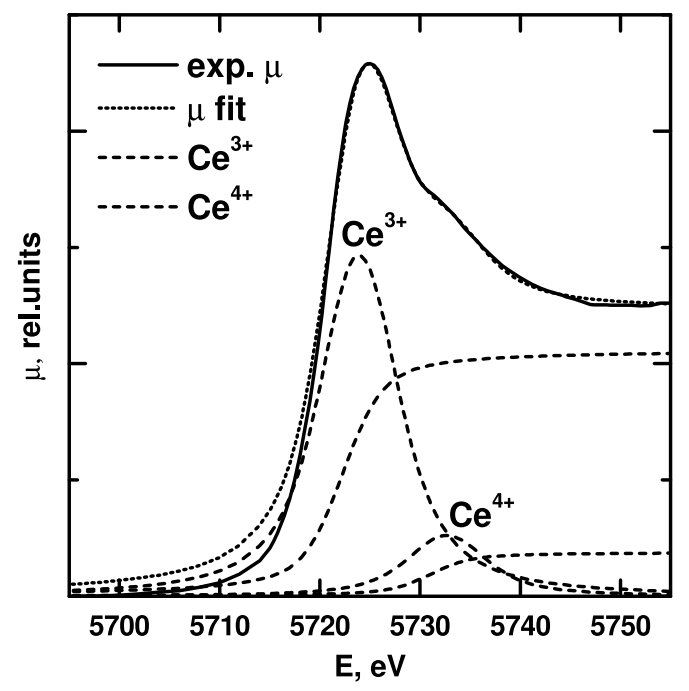

Fig. 3. Fitting of $\mathrm{Gd}_{0.85} \mathrm{Ce}_{0.15} \mathrm{Ni} \mathrm{Ce}_{3}$-XANES spectrum with the combination of Lorentzian and arctangent functions for the $\mathrm{Ce}^{3+}$ and $\mathrm{Ce}^{4+}$ contributions.

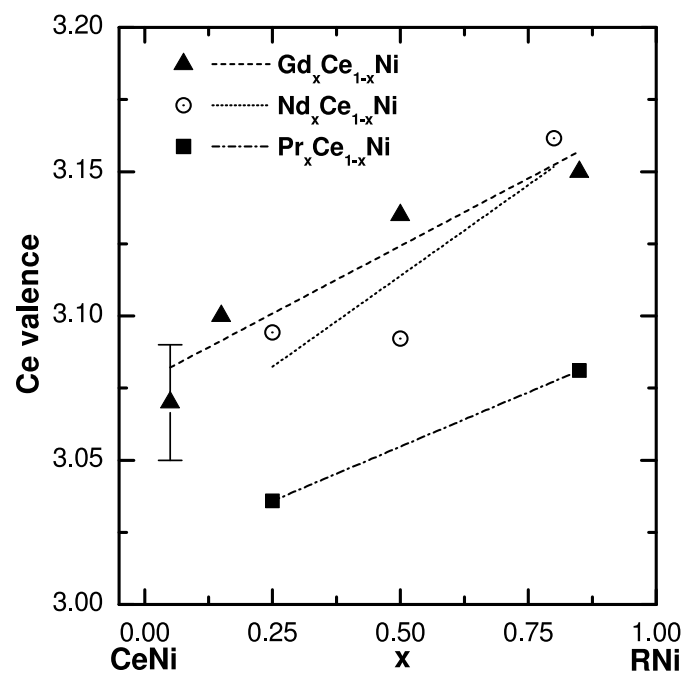

Fig. 4. Ce effective valence dependence upon the dopant concentration in the samples $(\mathrm{Gd}, \mathrm{Nd}, \mathrm{Pr})_{x} \mathrm{Ce}_{1-x} \mathrm{Ni}$ at room temperature.

agreement with the previous ones for similar systems [13]. For the lowest Gd dopant concentration $x=0.05$ the cerium effective valence in $\mathrm{Gd}_{x} \mathrm{Ce}_{1-x} \mathrm{Ni}$ is $\sim 3.07$. With a $\mathrm{Gd}$ concentration increase till $x=0.85$ the valence is increasing monotonously to $\sim 3.15$. Such a behavior of the cerium effective valence is determined by the difference between the ionic radii of cerium and gadolinium. The Gd dopant is characterized by a smaller ionic radius. Its insertion leads to the crystal-chemical compression of structure which causes delocalization of the $4 f$ states and an increase of the cerium effective valence. In the case of $\mathrm{Nd}_{x} \mathrm{Ce}_{1-x} \mathrm{Ni}$ the $\mathrm{Ce}$ valence changes from $\sim 3.09$ at $x=0.25$ to $\sim 3.16$ at $x=0.8$. The pronounced difference between the dependences for the Ce substitutions by $\mathrm{Gd}$ or $\mathrm{Nd}$ in the middle concentration range apparently arise from the larger ionic radius of neodymium which can not apply such a strong chemical pressure to the structure as gadolinium. The final $\mathrm{Ce}$ valence $\sim 3.16$ is similar for both dopants and might be the largest cerium effective valence obtainable in such CeNi-based compounds at room temperature. Praseodymium ions are of almost similar ionic radius as cerium ones and don't apply significant chemical pressure to the structure. Therefore, the cerium valence dependence in Pr-doped CeNi lies below the Gd and $\mathrm{Nd}$ curves. It is notable that in all compounds cerium never reaches the integer valence $3+$, hence the magnetic ordering should occur mainly in the Gd-, Pr-, Nd- substructures $[6,7]$.

The concentration dependence of the cerium valence in the $\mathrm{Ce}_{2} \mathrm{Fe}_{17-x} \mathrm{Mn}_{x}$ intermetallics at room temperature is shown in Fig. 5. In initial $\mathrm{Ce}_{2} \mathrm{Fe}_{17}$ the cerium effective valence is $\sim 3.34$ which is in good agreement with the data previously [14] obtained with the similar technique in another $\mathrm{Ce}_{2} \mathrm{Fe}_{17}$-based system. This value does not considerably change with the substitution of Fe by Mn. It is explainable as the total concentration of $\mathrm{Mn}$ in all compounds is not large in comparison with the Fe content, and hence there could be no significant change in the ionic interaction affecting the rare-earth ion. The larger value of the valence than in the CeNi-based samples is also consistent with the conception of ionic interactions 


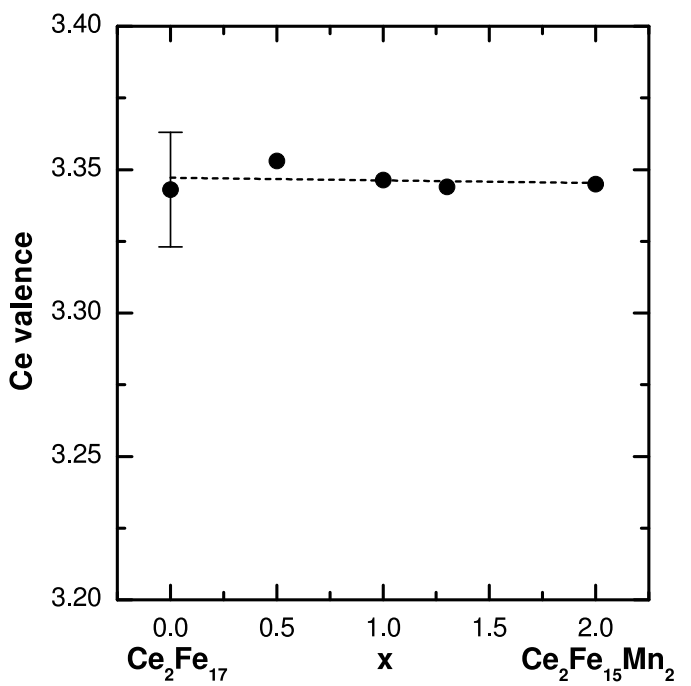

Fig. 5. Ce effective valence dependence upon the dopant concentration in $\mathrm{Ce}_{2} \mathrm{Fe}_{17-x} \mathrm{Mn}_{x}$ at room temperature.

between the rare-earth and $3 d$-metal. Its effect in CeNi is probably weaker because in the local enviroment of $\mathrm{Ce}$ in $\mathrm{CeNi}$ there are fewer $\mathrm{Ni}$ ions than $\mathrm{Fe}$ ions around $\mathrm{Ce}$ in $\mathrm{Ce}_{2} \mathrm{Fe}_{17}$. Besides, our results show that at room temperature there are no correlations between the magnetic ground state of the compounds $\mathrm{Ce}_{2} \mathrm{Fe}_{17-x} \mathrm{Mn}_{x}$ and the $\mathrm{Ce}$ valence fluctuations.

Because of the better components resolution the random error of the Ce valence determination in $\mathrm{Ce}_{2} \mathrm{Fe}_{17-x} \mathrm{Mn}_{x}$ is not more than 0.01. That is smaller than in the case of the CeNi-based compounds where the error could be $\sim 0.02$. So, this is the precision of the relative valence changes in corresponding dependences. However, the systematic error of the whole technique might be up to 0.03 .

The temperature dependences of the cerium effective valence in the compounds $\mathrm{Gd}_{x} \mathrm{Ce}_{1-x} \mathrm{Ni}$ and $\mathrm{Pr}_{x} \mathrm{Ce}_{1-x} \mathrm{Ni}$ of all stoichiometric compositions are shown in Figs. 6 and 7. In all Gd-doped compounds and also in $\operatorname{Pr}_{0.25} \mathrm{Ce}_{0.75} \mathrm{Ni}$, the cerium valence increases monotonously by $\sim 0.06$ with temperature decreasing from RT to $5 \mathrm{~K}$ and $10 \mathrm{~K}$, respectively. This corresponds to the gradual lattice compression

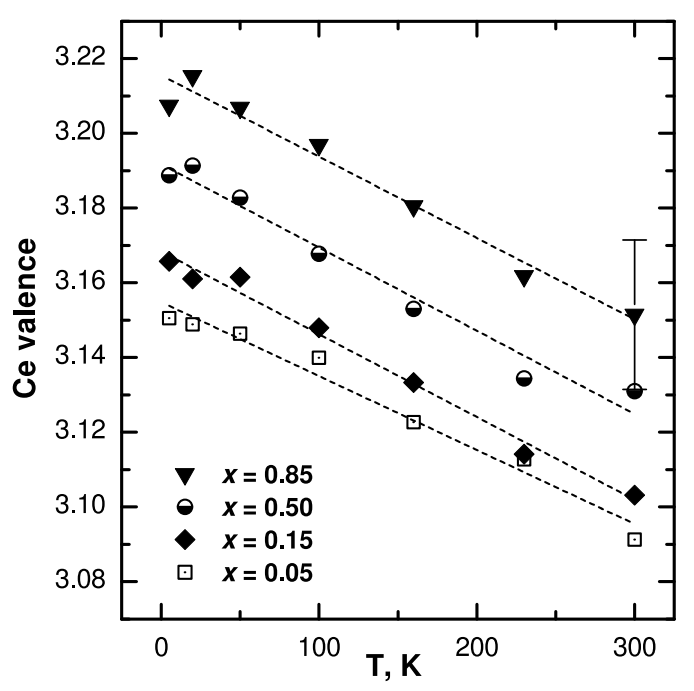

Fig. 6. Temperature dependences of the Ce effective valence in various $\mathrm{Gd}_{x} \mathrm{Ce}_{1-x} \mathrm{Ni}$ samples.

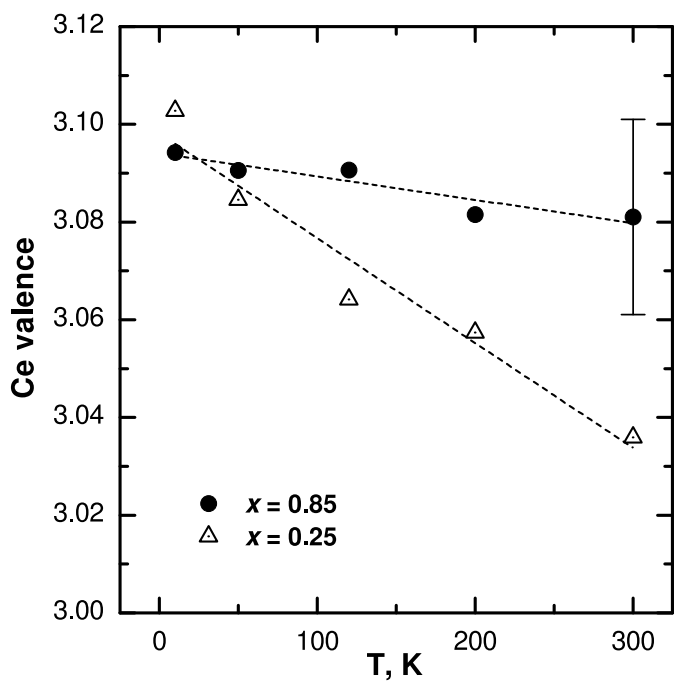

Fig. 7. Temperature dependences of the Ce effective valence in various $\operatorname{Pr}_{x} \mathrm{Ce}_{1-x} \mathrm{Ni}$ samples.

upon decreasing temperature. At all Gd concentrations the temperature change of the $\mathrm{Ce}$ valence probably is proportional to the same coefficient of the thermal expansion. However, the cerium valence in $\operatorname{Pr}_{0.85} \mathrm{Ce}_{0.15} \mathrm{Ni}$ does not significantly change with decreasing temperature. It increases slightly and within the error of the technique employed it might be considered as constant. Such a behavior of the cerium effective valence in $\operatorname{Pr}_{0.85} \mathrm{Ce}_{0.15} \mathrm{Ni}$ might be due to a large concentration of Pr ions which become the main ions forming the structure and possibly affect the coefficient of the thermal expansion, hence the lattice compresses weaker with decreasing temperature.

\section{Conclusion}

The cerium effective valence behavior in $(\mathrm{Gd}, \mathrm{Pr}, \mathrm{Nd})_{x} \mathrm{Ce}_{1-x} \mathrm{Ni}$ generally confirms the theory assuming the crystal-chemical compression to be the main driving force of the rare-earth ions valence state change. Whereas, the dependence of the Ce effective valence in $\mathrm{Ce}_{2} \mathrm{Fe}_{17-x} \mathrm{Mn}_{x}$ and the difference from the CeNi-based case do not contradict to the assumption that the presence of ionic components in the interatomic interaction might influence the rare-earth valence in compounds with $3 d$-metals.

In all CeNi-based compounds cerium is in an intermediate valence state and the magnetic ordering really should occur generally in the dopant's substructure [6, 7]. While the assumption about the connection of the magnetic properties and the Ce valence state in $\mathrm{Ce}_{2} \mathrm{Fe}_{17-x} \mathrm{Mn}_{x}$ intermetallics seems to be disproved at least at room temperature by the absence of correlations between the type of the magnetic state and the valence dependence. For the better clarification of this connection XAFS studies at low temperatures and also with magnetic circular dichroism are desirable.

Acknowledgments. This work was partially supported by RFBR (grants No. 08-02-00759-a and 09-02-12257-ofi_m), Federal target program "Scientific and scientific-pedagogical stuff of innovative Russia" (State Contracts No. P2597 and P39) and UD RAS (project No. 09-P-2-1008). 


\section{References}

[1] Khomskii, D. I.: The problem of intermediate valence. PhysicsUspekhi. 129(3) (1979) 443-485.

[2] Johansson, B.: The $\alpha-\gamma$ Transition in Cerium is a Mott Transition. Phil. Mag. 30 (1974) 469-479.

[3] Isnard, O.; Miraglia, S.; Giorgetti, C.; Dartyge, E.; Krill, G.; Fruchart, D.: Ce valence state probed by XAFS study in $\mathrm{Ce}_{2} \mathrm{Fe}_{17-x} \mathrm{Ga}_{x} \mathrm{H}_{y}$ compounds. J. Alloys Compds. 262/263 (1997) 198-201.

[4] Lukoyanov, A. V.; Shkvarin, A. S.; Knyazev, Yu. V.; Kuz'min, Yu. I.; Kuchin, A. G.; Efremova, N. N.; Finkelshtein, L. D.; Nekrasov, I. A.: Electronic structure of $\mathrm{Ce}_{2} \mathrm{Fe}_{17}$ and $\mathrm{Ce}_{2} \mathrm{Fe}_{15.3} \mathrm{M}_{1.7}$ $(\mathrm{M}=\mathrm{Al}$ and $\mathrm{Si}$ ) intermetallics: experiment and theory. Phys. Solid State. 49 (2007) 95-101.

[5] Bobet, J.-L.; Grigorova, E.; Chevalier, B.; Khrussanova, M.; Peshev, P.: Hydrogenation of CeNi: hydride formation, structure and magnetic properties. Intermetallics. 14(2) (2006) 208-212.

[6] Clementyev, E. S.; Mirmelstein, A. V.; Alekseev, P. A.; Lazukov, V. N.; Gribanov, A. V.; Lapertot, G.: Enhancement of localized magnetism due to Kondo ions. Proc. of Int. Symposium "Phase transitions in alloys" OMA-12, Loo. 2 (2009) 78.

[7] Mirmelstein, A.; Clementyev, E.; Lapertot, G.: Enhancement of localized magnetism due to Kondo ions. 6th US-Russian $\mathrm{Pu}$ science Workshop, Livermore (2006) 56-57.
[8] Givord, D.; Lemaire, R.: Magnetic transition and anomalious thermal expansion in $\mathrm{R}_{2} \mathrm{Fe}_{17}$ compounds. IEEE Trans. Magn. MAG-10(2) (1974) 109-113.

[9] Kuchin, A. G.; Pirogov, A. N.; Khrabrov, V. I.; Teplykh, A. E.; Ermolenko, A. S.; Belozerov, E. V.: Magnetic and structural properties of $\mathrm{Ce}_{2} \mathrm{Fe}_{17-x} \mathrm{Mn}_{x}$ compounds. J. Alloys Compds. 313 (2000) 7-12.

[10] Ankudinov, A. L.; Bouldin, C.; Rehr, J. J.; Sims, J.; Hung, H.: Parallel calculation of electron multiple scattering using Lanczos algorithms. Phys. Rev. B 65 (2002) 104107-1-104107-11.

[11] Röhler, J.: LIII-absorption on valence fluctuating materials. J. Magn. Magn. Mater. $47 / 48$ (1975) 175-180.

[12] Krause, M. O.; Oliver, J. H.: Natural widths of atomic K and L levels, $K_{\alpha}$ X-ray lines and several KLL auger lines. J. Phys. Chem. Rev. Data. 8(2) (1979) 329-338.

[13] Alekseev, P. A.; Clementyev, E. S.; Lazukov, V. N.; Nefedova, E. V.; Sadikov, I. P.; Efremova, N. N.; Finkelshtein, L. D.; Kolchugina, N. B.; Chistiakov, O. D.: The closest environment influence on the $\mathrm{Ce}$ ions valence in the compounds of $\mathrm{RNi}_{5}$ and RNi type. Phys. Met. Metalloved. 77(6) (1994) 60-68.

[14] Isnard, O.; Miraglia, S.; Fruchart, D.; Giorgetti, C.; Dartyge, E.; Krill, G.: X-ray absorption spectroscopy and magnetic circular $\mathrm{X}$-ray dichroism in $\mathrm{Ce}_{2} \mathrm{Fe}_{17} \mathrm{~N}_{3}$. Condens. Matter. 8 (1996) 2437-2446. 